\title{
Holographic Origin of Missing Mass and Cosmological Constant Related to Mass Defect in Semiclosed Friedman Universe
}

\author{
Noboru Hokkyo \\ Senjikan Institute, Niigata, Japan \\ Email: noboruhok@yahoo.co.jp
}

Received 11 November 2014; revised 13 December 2014; accepted 29 December 2014

Copyright (C) 2014 by author and OALib.

This work is licensed under the Creative Commons Attribution International License (CC BY). http://creativecommons.org/licenses/by/4.0/

(c) (†) Dpen Access

\section{Abstract}

A holographic origin of missing mass and extremely high value of Einstein's cosmological constant $\Lambda \sim 10^{122}$ in quantum cosmology, compared to observational data $0 \leq \Lambda \leq 1$, is sought and related to extremely large relativistic mass defect of gravitional semiclosure of Friedman universe joined to an asymptotically flat outer space.

\section{Keywords}

\section{Cosmology, General Relativity, Quantum Theory, Holography, Blackhole, Semiclosed Friedman Universe}

Subject Areas: Quantum Mechanics, Special Theory of Relativity

\section{Introduction}

Inflationary cosmology succeeded in explaining the origin of the large-scale structure of observed universe evolving from a singular Big Bang or large quantum fluctuations of pre-existing spacetime metric in causally related small region, answering why the present universe appears flat, homogeneous and isotropic. Yet, apart from problems of the fine tuning of initial conditions and the unitarity of the expansion history [1]-[3], there have been continued interpretational controversies regarding the extremely high cosmological constant in quantum cosmology. Here its holographic origin is sought and related to extremely large relativistic mass defect of gravitional semiclosure of Friedman universe joined to an asymptotically flat outer space.

\section{Dimensional Cosmological Constant}

The Hubble parameter $H$ was originally used to represent Hubble law, $v=H d$, relating the relative velocity $v$

How to cite this paper: Hokkyo, N. (2014) Holographic Origin of Missing Mass and Cosmological Constant Related to Mass Defect in Semiclosed Friedman Universe. Open Access Library Journal, 1: e1233. http://dx.doi.org/10.4236/oalib.1101233 
of the extragalactic objects at a distance $d$ receding away from the Earth. In the Friedman universe $H$ is defined as $H(t)=(\mathrm{d} a(t) / \mathrm{d} t) / a(t)$, where $a(t)$ is a scale factor normalized to $a=1$ at the present epoch $t=t_{0} \sim 14 \mathrm{Gyr}$, and obeys the equation [2]

$$
H(t)^{2}=\frac{8 \pi G}{3}\left[\rho_{m 0} / a(t)^{3}+\rho_{r 0} / a(t)^{4}+\rho_{\Lambda 0} / a(t)^{3(\omega+1)}\right],
$$

here $\rho_{m 0}$ is the nonrelativistic matter (dark and luminous) density, $\rho_{r 0}$ the relativistic radiation density, and $\rho_{\Lambda 0}$ the dark energy density at $t=t_{0}$ where $\omega=p_{\Lambda} c^{2} / \rho_{\Lambda}<-1\left(\rho_{\Lambda}+p_{\Lambda} c^{2}<0\right)$ is the equation of state relating $\rho_{\Lambda}$ and pressure $p_{\Lambda} c^{2}$ in inflationary cosmology. The most direct evidence of dark energy comes from observations of supernpovae with uniform energy density.

In the Friedman-Lemaître cosmology the expansion history is determined by a set of dimensionless parameters at present epoch whose sum is normalized to uinity. As the observed energy density in the cosmic background radiation show $S$ a minor contribution $\Omega_{r} \sim 1 \times 10^{-4}$ to $H_{0}^{2}$ the following numerical relations between dimensionless density parameters $\Omega_{\Lambda, m}$ and the critical densities $\rho_{c \Lambda, m, 0}$ are considered significant [2]:

$$
\Omega_{\Lambda}+\Omega_{m}=1 \text {, }
$$

where $\Omega_{\Lambda}=8 \pi \rho_{\Lambda} G / 3 H_{0}^{2}=\rho_{\Lambda} / \rho_{c \Lambda 0}=0.72$ and $\Omega_{m}=8 \pi \rho_{m} G / 3 H_{0}^{2}=\rho_{m} / \rho_{c m 0}=0.28$.

Only from dimensional consideration we put $\Lambda=c^{5} / \hbar G=c^{2} / l_{p l}^{2} \sim 10^{88} \mathrm{sec}^{-2}$, where $\hbar=1.026 \times 10^{-27}$ erg-sec is the Planck constant and $l_{p l}=c^{2} / \hbar G \sim 10^{-33} \mathrm{~cm}$ the Planck length. Using the cosmological unit $h=0.5-0.9$ defined in $H_{0}=100 \mathrm{hkm} / \mathrm{sec} \cdot \mathrm{Mpc}$ in observational cosmology, we get

$$
\Lambda / H_{0}^{2} \sim 10^{122} \mathrm{~h}^{-2} .
$$

\section{Holographic Cosmological Constant}

The holographic principle in string theory [4] states that the description of events in a volume of spacetime can be encoded on the boundary to the region like a gravitational horizon. The principle suggests that the entire universe can be seen as a two-dimensional information structure on the cosmological horizon with possible quantum fluctuations. From the observed cosmic back-ground microwave temperature $T=2.7 \mathrm{~K}$ the entropy density $s$ of the universe at $t=t_{0}$ is estimated by $s \sim g T^{3}$. Using $g=2$ for photon we have $s_{\gamma}\left(t_{0}\right) \sim 1.5$. A volume estimate $V=(4 \pi / 3) R^{3}$ with $R=10^{28} \mathrm{~cm}$ gives a total radiation entropy $S_{\gamma} \sim 6.3 \times 10^{8 \gamma^{\gamma}}$. The entropy contribution from baryons is smaller than $S_{\gamma}$. Inclusion of neutrino contribution increases $S_{\gamma}$ to $S_{\gamma+v} \sim 10^{88}$. This is well below the holographic bound of the present universe dictated by the area in terms of the Planck units $l_{p l}$ giving

$$
S_{\text {holog }}\left(\mathrm{t}_{0}\right) \sim\left(R / l_{p l}\right)^{2} \sim 10^{122} .
$$

It is suggested [2] that 34 orders of magnitude difference between $S_{\gamma+v}$ and $S_{\text {holog }}$ comes from extragalactic blackholes. We recall that a typical stellar obeject having mass [4]

$$
M \sim m_{p l}\left(l_{p} / l_{p l}\right)^{2},
$$

has a holographic information content $\left(l_{p} / l_{p l}\right)^{2}=\left(10^{-24} / 10^{-33}\right)^{2} \sim 10^{20}$ where $l_{p}$ is the proton wavelength.

\section{Mass Defect in Semiclosed Universe}

The possibility of joining-on of the dust-filled semi-closed Friedman universe to an asymptotically flat space through Schwarzschid throat (Einstein-Rosen bridge) was first pointed out by Tolman [5] and used by Oppenheimer and Snyder [6] in their study of the dust motion in massive stellar objects, followed by Milne [7], Zel'dovich [8] and Novikov [9] in the form extendible to the electrically charged universe joined onto flat outer space through double-valued Reissner-Nordström bottleneck prevented from gravitational pinch-off by the gauge field lines of force extending to infinity or onto an oppostely charged anti-universe. While Novikov considered quasistellar radio sources as parts of the semiclosed Friedman universe, each delayed in expansion beyond their Schwarzschild horizon with matter falling in from outside, Zel'dovich asked: Do there exist in nature objects of the type of the semiclosd world? Can such objects be formed from rarefied matter in an open world in 
an finite world time? And wrote: The possibility of their existence cannot be excluded for Friedman universe with small energy density and large mass defect.

Consider the radial line element $\mathrm{d} s=c \mathrm{~d} \tau$

$$
\mathrm{d} s^{2}=c^{2} \mathrm{~d} t^{2}-g_{r r} \mathrm{~d} r^{2}, \quad g_{r r}=\left(1-\left(r / r_{g}\right)^{2}\right)^{-1},
$$

where $\tau$ is the proper time and $r_{g}=\frac{3 c^{2}}{8 \pi G \rho_{\Lambda}}$ is the gravitional radius determining the cosmological horizon. The proper volume $V_{p}$ and the mass $M_{p}$ of the universe are given as

$$
M_{p}=\rho_{\Lambda} V_{p}=2 \pi \rho_{\Lambda} \int R_{r^{2}} g_{r r} \mathrm{~d} r=(3 / 2)\left(R / r_{g}\right)^{3}\left[\sin ^{-1}\left(R / r_{g}\right)-\left(R / r_{g}\right)\left(1-\left(R^{2} / r_{g}^{2}\right)^{1 / 2}\right)\right] M,
$$

where $M=\rho_{\Lambda} V=4 \pi \rho_{\Lambda} R^{3} / 3$ is the Newtonian mass. We call $\Delta M=M_{p}-M$ the mass defect. As the world radius $r$ increases from $r=l_{p l}$, where $\Omega_{\Lambda}=V_{p} / V \sim 0$ and $\sin ^{-1}\left(R / r_{g}\right) \sim 0$, the proper radius $R_{p}=\int R_{r^{2}} g_{r r} \mathrm{~d} r$ increases until its maximum value $R$ is reached at $\Omega_{\Lambda}=V_{p} / V=0.5$, where $\sin ^{-1}\left(R / r_{g}\right)=\pi / 2, M_{p}=0.5$ and $\Delta M=0$. With further increase of $r, R_{p}$ decrease towards $R_{p}=0$ at $\Omega_{\Lambda}=V_{p} / V=0$, where $\sin ^{-1}\left(R / r_{g}\right)=\pi$ and $M_{p}=0$, forming a gravitational self-closure or semi-closure having surface area $l_{p l}^{2}$ creating Planck scale blackholes or quantm flucuations in asymptotically flat outer space [1]. We note that $\Delta M>0$ during expansion, $0<\Omega_{\Lambda}<0.5$, of the universe and $\Delta M<0$ during contraction, $0.5<\Omega_{\Lambda}<1$. The informational content of the universe is thought [1] [2] to increase by the factor $\left(R / l_{p l}\right)^{2} \sim 10^{122}$ during expansion and decreases by the factor $\left(l_{p l} / R\right)^{2} \sim 10^{-122}$ during contraction so that

$$
\Lambda_{\text {holog }}=\left(l_{p l} / R\right)^{2} \Lambda=10^{-122} \Lambda \text { for } 0.5<\Omega_{\Lambda}<1 .
$$

We note that the equation of state $\rho_{\Lambda}<0$ required by the dark energy is satisfied for $0.5<\Omega_{\Lambda}<1$ where $\Delta M<0$. There the negative gravitional attractive energy between $\rho_{\Lambda}$ exceeds the added rest energy $\rho_{\Lambda}$.

\section{Conclusion}

We have seen that the extremely high value of the cosmological constant $\Lambda \sim 10^{122}$ can be suppressed to $\Lambda_{\text {holog }} \sim 1$ by the mass defect or missing mass $\Delta M<0$ in the upper hemisphere, $0.5<\Omega_{m}<1$, of the semiclosed Friedman universe where the observation indicates $\Omega_{\Lambda}=0.72$, with probable creation of extragaractic blackholes for $\Omega_{\Lambda}=1$. The stellar objects filled with baryonic dust are likely to occupy the lower hemisphere, $0<\Omega_{m} \leq 0.5$, where the observation indicates $\Omega_{m}=0.28$.

\section{References}

[1] Hawking, S.W. (1984) The Cosmological Constant Is Probably Zero. Physics Letters B, 134, 403-404. http://dx.doi.org/10.1016/0370-2693(84)91370-4

[2] Baum, L. and Frampton, P.H. (2007) PRL, 98, 07130.

[3] Weinberg, S. (1972) Gravitation and Cosmology. John Wiley \& Sons, New York.

[4] Witten, E. (1984) Some Properties of O(32) Superstrings. Physics Letters B, 149, 351-356. http://dx.doi.org/10.1016/0370-2693(84)90422-2

[5] Tolman, R.C. (1934) Effect of Inhomogeneity on Cosmological Models. Proceedings of the National Academy of Sciences, 20, 169. http://dx.doi.org/10.1073/pnas.20.3.169

[6] Oppenheimer, J. and Snyder, H. (1039) 56, 445.

[7] Milne, E. (1942) On “Absolute Acceleration”. Nature, 150, 489. http://dx.doi.org/10.1038/150489a0

[8] Zel'dovich, Ya.B. (1962) J. Exptl. Theoret. Phys. U.S.S.R, 43, 19.

[9] Novikov, I.D. (1963) Soviet Astron.-A. J., 7, 537

[10] Chandrasekhar, S. (1985) The Mathematical Theory of Black Holes. 